\title{
The Brief Symptom Inventory and the Outcome Questionnaire-45 in the Assessment of the Outcome Quality of Mental Health Interventions
}

\author{
Aureliano Crameri, ${ }^{1}$ Christopher Schuetz, ${ }^{2}$ Andreas Andreae, ${ }^{2}$ Margit Koemeda, ${ }^{3}$ \\ Peter Schulthess, ${ }^{3}$ Volker Tschuschke, ${ }^{4,5}$ and Agnes von $\mathrm{Wyl}^{1}$ \\ ${ }^{1}$ Zurich University of Applied Sciences, Zurich, Switzerland \\ ${ }^{2}$ Integrated Psychiatric Services Winterthur and Zurich Unterland (ipw), Winterthur, Switzerland \\ ${ }^{3}$ Swiss Charter of Psychotherapy, Stäfa, Switzerland \\ ${ }^{4}$ University Hospital of Cologne, Cologne, Germany \\ ${ }^{5}$ Sigmund Freud University, Berlin, Germany \\ Correspondence should be addressed to Aureliano Crameri; aureliano.crameri@zhaw.ch
}

Received 2 June 2016; Accepted 11 August 2016

Academic Editor: Yvonne Forsell

Copyright ( $\odot 2016$ Aureliano Crameri et al. This is an open access article distributed under the Creative Commons Attribution License, which permits unrestricted use, distribution, and reproduction in any medium, provided the original work is properly cited.

\begin{abstract}
Self-report questionnaires are economical instruments for routine outcome assessment. In this study, the performance of the German version of the Outcome Questionnaire-45 (OQ-45) and the Brief Symptom Inventory (BSI) was evaluated when applied in analysis of the outcome quality of psychiatric and psychotherapeutic interventions. Pre-post data from two inpatient samples $(N=5711)$ and one outpatient sample $(N=239)$ were analyzed. Critical differences (reliable change index $)$ and cut-off points between functional and dysfunctional populations were calculated using the Jacobson and Truax method of calculating clinical significance. Overall, the results indicated that the BSI was more accurate than the OQ-45 in correctly classifying patients as clinical subjects. Nonetheless, even with the BSI, about $25 \%$ of inpatients with schizophrenia attained a score at admission below the clinical cut-off. Both questionnaires exhibited the highest sensitivity to psychopathology with patients with personality disorders. When considering the differences in the prescores, both questionnaires showed the same sensitivity to change. The advantage of using these self-report measures is observed primarily in assessing outpatient psychotherapy outcome. In an inpatient setting two main problems - namely, the low response rate and the scarce sensitivity to psychopathology with severely ill patients-limit the usability of self-report questionnaires.
\end{abstract}

\section{Introduction}

Along with accessibility to services, appropriateness of treatment, and perception of care, outcome is an important quality indicator in mental health care [1]. A widely used approach to assess outcome quality is measuring symptom reduction through rating scales, either clinician-administered or selfreported. Self-report scales are widely used in outcome evaluation of psychotherapies [2]. Their standardized form allows easy administration by clinicians without additional training and also guarantees a high level of reliability. On the other hand, for patients with a high degree of psychopathology, clinician-administered scales are preferred [3], for with these scales, the psychological status of each patient can be assessed independently of the person's capability or willingness to accurately describe their relevant symptoms and behaviors. However, achieving good rating quality requires clinician training in the application of these instruments plus subsequent assessment of interexaminer reliability. Given that in general psychiatric hospitals it has been proven to be quite difficult to routinely involve clinicians in training courses, the question arises as to whether clinician-rated assessments can be substituted by similar scales filled out by psychotherapy patients themselves (self-report) [4]. 
For general assessment of mental health symptoms, one of the frequently suggested multidimensional self-report instruments is the Symptom Checklist (SCL-90R) or its short form, the Brief Symptom Inventory (BSI) [5-7]. However, based on the number of published studies implementing these questionnaires, it can be asserted that in the field of mental health they have been used predominantly with outpatients and far less frequently with inpatients or, more generally, with patients with severe mental illness [8-10]. Nevertheless, these instruments seem promising in assessing the mental health status of the latter patient group, because they also contain specific scales for measuring psychotic and schizotypal symptoms, such as the Psychoticism or the Paranoid Ideation scale. However, the validity of these scales has not as yet been unequivocally confirmed. Wood [11] found no evidence that patients with schizophrenia score higher on the Psychoticism scale than patients without schizophrenia. Johnson et al. [12] found no differences on the Paranoid Ideation and Psychoticism scales between patients diagnosed with or without schizophrenia. In contrast, Preston and Harrison [13] demonstrated in a sample of 69 patients presenting their first psychotic episode that responses on the BSI items could discriminate between patients with weak and with marked positive symptoms.

Another instrument recommended for assessment of individuals seeking mental health treatment is the Outcome Questionnaire-45 (OQ-45) [14-16]. It is a newer, selfreport outcome measure created primarily for psychotherapy patients, which has already been used in psychiatric inpatient care $[17,18]$.

In the context of quality assurance programs, quantitative data collected via mental health questionnaires find their application in the analysis of pre-post differences, which reflect the intraindividual changes attained during the treatment. One widely used analysis method is the calculation of clinical significance proposed by Jacobson and Truax [19]. The aim of the method is to identify which patients move outside the range of the dysfunctional population and consequently attain "normal" functioning. Patients who improve significantly and cross a well-defined cut-off score between dysfunctional and functional distributions are classified as "remitted." However, patients can only be consistently classified in this way if the self-report measurement at the beginning of the treatment correctly identifies them as dysfunctional.

The objective of this study was to evaluate the German versions of the OQ-45 and the BSI when applied in the analysis of outcome quality of inpatient and outpatient treatments. The analyses focused on three aspects of usability:

(1) Response Rate. How many patients fill out the questionnaires? To produce a representative outcome estimation, the majority of the patients should be able to fill out the self-report forms. A high rate of nonresponse increases the risk of bias in the results.

(2) Sensitivity to Psychopathology. Applying the cutoff scores calculated with the Jacobson and Truax method, what percentages of patients are correctly classified by the self-report measures at admission as belonging to the dysfunctional population?

(3) Sensitivity to Change. Do the two questionnaires have the same sensitivity to change? The equivalence of the two measures is not obvious, since compared to the BSI, the OQ-45 contains fewer symptom-specific items and focuses partially on social functioning (e.g., work), which requires more time for change to occur than the time required to see change in acute symptoms.

\section{Methods}

2.1. Samples. Three different samples, two inpatient samples and one outpatient sample, were used for this study. Both inpatient samples were treated at an inpatient clinic in Switzerland, the Integrated Psychiatric Services of Winterthur, and Zurich Unterland (ipw).

At the ipw, the OQ-45 was implemented as a self-report measure of outcome quality from 2008 to 2010; it was replaced by the BSI starting in 2012. For samples 1 and 2, inpatient treatments from 2008 to 2009 (OQ-45) and from 2012 and 2013 (BSI) were selected from the database using the following inclusion criteria:

(i) A principal diagnosis belonging to one of these major groups: F1 (substance abuse), F2 (schizophrenia or other psychotic disorders), F3 (mood disorders), F4 (anxiety or stress related disorders), and F6 (personality disorders).

(ii) Age of at least 18 years.

(iii) Hospitalization of at least 7 days.

Sample 3 was recruited in a project on outpatients promoted by the Swiss Charta for Psychotherapy. The aim of this nonrandomized field study is to investigate various processoutcome aspects of outpatient treatments carried out with different experiential or psychodynamic therapy methods [26-28]. Cases from this sample were selected using the following criteria:

(i) At least one Axis I disorder according to the criteria of DSM-IV.

(ii) Age of at least 18 years.

(iii) Minimum of 10 treatment sessions.

Both studies above were carried out in accordance with the ethical principles of the Declaration of Helsinki. For the Swiss Charta project, a research application was submitted to the ethics committee of each of the Swiss cantons in which the projects were carried out; all of the applications were approved. All patients gave informed written consent for their participation in the study. Data from the ipw were collected within an internal quality measurement system for which, according to Swiss federal and cantonal law, no ethical approval was required. For this reason, the present study was granted an exemption from the requirement for ethics approval by the Cantonal Ethics Committee of Zurich 
TABLE 1: Descriptive statistics of the analyzed samples.

\begin{tabular}{|c|c|c|c|}
\hline & $\begin{array}{c}\text { Sample 1 } \\
\left(N_{1}=2894\right)\end{array}$ & $\begin{array}{c}\text { Sample } 2 \\
\left(N_{2}=2877\right)\end{array}$ & $\begin{array}{c}\text { Sample } 3 \\
\left(N_{3}=239\right)\end{array}$ \\
\hline \multicolumn{4}{|l|}{ Sex } \\
\hline Female & $1424(49.2 \%)$ & $1532(53.2 \%)$ & $162(67.8 \%)$ \\
\hline Male & $1470(50.8 \%)$ & $1345(46.8 \%)$ & $77(32.2 \%)$ \\
\hline \multicolumn{4}{|l|}{ Education } \\
\hline Low & $1005(34.7 \%)$ & $895(31.1 \%)$ & $24(10.0 \%)$ \\
\hline Middle & $1461(50.5 \%)$ & $1488(51.7 \%)$ & $86(36.0 \%)$ \\
\hline High & $428(14.8 \%)$ & $494(17.2 \%)$ & $129(54.0 \%)$ \\
\hline \multicolumn{4}{|l|}{ Income } \\
\hline Salary & $729(25.2 \%)$ & $687(23.9 \%)$ & $185(77.4 \%)$ \\
\hline Sickness/disability benefit & $1060(36.6 \%)$ & $1147(39.9 \%)$ & $23(9.6 \%)$ \\
\hline Social welfare payments & $425(14.7 \%)$ & $373(13.0 \%)$ & $3(1.3 \%)$ \\
\hline Old age insurance & $232(8.0 \%)$ & $292(10.1 \%)$ & $7(2.9 \%)$ \\
\hline Other & $448(15.5 \%)$ & $378(13.1 \%)$ & $21(8.8 \%)$ \\
\hline GAF & $47.0(17.2)$ & $38.5(14.3)$ & $60.5(13.1)$ \\
\hline \multirow{5}{*}{ Principal diagnosis (ICD-10/DSM-IV) } & F1: $884(30.6 \%)$ & F1: $594(20.6 \%)$ & Mood: 107 (44.8\%) \\
\hline & F2: $681(23.5 \%)$ & F2: $649(22.6 \%)$ & Anxiety: 65 (27.2\%) \\
\hline & F3: $706(24.4 \%)$ & F3: $927(32.2 \%)$ & Adjustment: 45 (18.8\%) \\
\hline & $\mathrm{F} 4: 386(13.3 \%)$ & F4: $461(16.0 \%)$ & Other: $22(9.2 \%)$ \\
\hline & F6: $237(8.2 \%)$ & F6: $246(8.6 \%)$ & \\
\hline Duration of treatment & 36.7 days (38.1) & 36.0 days (31.7) & 41.3 sessions $(34.2)$ \\
\hline \multicolumn{4}{|l|}{ Type of discharge } \\
\hline Mutual consent & $2327(80.4 \%)$ & $2489(86.5 \%)$ & $180(75.3 \%)$ \\
\hline Decided by the patient & $221(7.6 \%)$ & $117(4.1 \%)$ & $46(19.2 \%)$ \\
\hline Decided by the treating person & $215(7.4 \%)$ & $181(6.3 \%)$ & $11(4.6 \%)$ \\
\hline Other & $131(4.6 \%)$ & $90(3.1 \%)$ & $2(0.1 \%)$ \\
\hline
\end{tabular}

(Waiver number 09-2016). Participating patients at the ipw gave informed verbal consent for the use of the data.

Table 1 presents the characteristics of the three samples. In the inpatient samples 1 and 2 the most frequent substancerelated disorder (F1) was associated with alcohol consumption $(71.5 \%$ of the patients with an F1 diagnosis in sample 1 and $56.2 \%$ in sample 2, resp.). In the F2 group paranoid schizophrenia was prominent $(61.8 \%$ and $59.3 \%$, resp., of the F2 group). Most of the patients with a mood disorder had either a depressive episode $(53.2 \%$ and $51.0 \%$ within the F3 group) or a recurrent depressive disorder $(40.3 \%$ and $45.4 \%$ within the F3 group). The most frequently diagnosed personality disorder was of the Borderline type $(64.6 \%$ and $53.6 \%$ within the F6 group). In the outpatient setting, $69.2 \%$ of diagnosed mood disorders were major depressions. The most frequent anxiety disorders in this setting were social phobia, panic disorder/agoraphobia, and generalized anxiety disorder.

2.2. Measures. The following self-report and clinicianadministered instruments were used for this analysis:

(i) Basic Documentation. Important clinical information and sociodemographic characteristics were recorded on a form. These data were collected by the treating psychiatrists (for inpatients) and the treating psychotherapists (for outpatients).

(ii) ICD-10. Diagnoses based on this system were available for the inpatient samples and had been assigned by the treating psychiatrists.

(iii) Structured Clinical Interviews for DSM-IV (SCID-I/II) [29]. These interviews, which were available only for sample 3, served in assessing Axis I and II disorders (clinical syndromes and personality disorders) according to the DSM-IV criteria. Trained psychologists (not involved in treatment of the patients) were engaged to carry out the two clinical interviews.

(iv) Clinical Global Impressions (CGI) [30]. This instrument gathers the clinician's view of the severity of psychopathology (CGI-S) and of the improvements from the initiation point of the treatment (CGI-I). The two aspects are each rated on a 7-point scale. Ratings with this instrument were available only for the inpatient samples and were provided by the treating psychiatrists. 
(v) Global Assessment of Functioning (GAF) [31]. On this scale psychological, social, and occupational functioning are rated on a hypothetical continuum from severe mental illness (0) to mental health (100).

(vi) OQ-45. The questionnaire consists of 45 items grouped in the three scales Symptom Distress (SD), Interpersonal Relations (IR), and Social Role (SR), which add up to a total score (OQ Total Score). The scale structure of the original version is supported by confirmatory factor analysis [14]. The single scales in the German version exhibit good internal consistency [20].

(vii) BSI. This questionnaire is the short version of the SCL-90R [6]. The 53 items assess nine primary symptom dimensions: Somatization (SOM), Obsessive-Compulsiveness (OBS), Interpersonal Sensitivity (INS), Depression (DEP), Anxiety (ANX), Hostility (HOS), Phobic Anxiety (PHOB), Paranoid Ideation (PAR), and Psychoticism (PSY). Additional global indices of distress can be obtained, for example, the General Symptom Index (GSI), which is the mean score of all items. In the analysis carried out by Derogatis and Melisaratos [5] seven of the primary scales showed a clear convergence with their counterparts among the MMPI scales. All scales in the German version exhibit satisfactory test-retest reliability [22].

\subsection{Statistical Analysis}

2.3.1. Missing Data. Nonresponse is a common problem in surveys and can occur for single items or for the entire examination. Analyzing only data of patients that responded to every item on the self-report measures at admission as well as at discharge would lead to a substantial loss of information, especially for the analyses for the inpatient setting. To include as many cases as possible, we defined different inclusion criteria for each analysis (Figure 1). First, we defined the minimal number of answered items to calculate scale scores. In a previous unpublished study we tested (through simulations) the impact of incomplete item values in the calculation of scores for the OQ-45 scales. Our results showed that if, for the three scales, namely, SD, IR, and SR, at least 8,6 , and 6 items, respectively, had a valid value, then the generated scale scores preserved a correlation of $r>0.90$ with the corresponding scores based on completely observed values. In this study, we considered as evaluable only data records that fulfilled the rule mentioned above. Concerning the BSI, we analyzed only returned questionnaires with at least 40 valid item values [32].

We calculated the response rate on the basis of the number of returned questionnaires that fulfilled the abovementioned criteria of completeness. Patients who completed a sufficient number of items at admission were included in the analysis of sensitivity to psychopathology. Patients who additionally did the same at discharge were included in the analysis of sensitivity to change.
2.3.2. Parameters of Clinical Significance. For the analysis of intraindividual changes we applied Jacobson and Truax's method of calculating clinical significance [19], with which the proportions of improved and recovered cases can be calculated [2]. To determine these proportions, two parameters are needed: (1) a critical difference $D$, which allows identification of individual pre-post differences that are sufficiently large to be considered statistically significant (reliable change) and (2) a cut-off $C$, which distinguishes scores that are a variation of normal functioning from scores indicating a psychopathological state. Therefore, patients exhibiting a reduction of at least $D$ points from their initial impairment score are considered "improved." If their score additionally falls below the cut-off $C$, then they are considered "remitted."

The critical difference, for example, at a confidence level of $95 \%$, is obtained by using the standard deviation and the reliability coefficients of the measure:

$$
D_{95 \%}=1.96 \cdot \mathrm{SD}_{\text {patient }} \cdot \sqrt{2(1-r)},
$$

whereas the cut-off is calculated as a weighted midpoint between the means of a patient and a nonpatient population as follows:

$$
C=\frac{\mathrm{SD}_{\text {patient }} \cdot M_{\text {nonpatient }}+\mathrm{SD}_{\text {nonpatient }} \cdot M_{\text {patient }}}{\mathrm{SD}_{\text {patient }}+\mathrm{SD}_{\text {nonpatient }}} .
$$

We additionally compared our parameter values with those published in other studies on the basis of samples from Germany.

2.3.3. Outcome Comparisons. To compare sensitivity to change, measured with two different questionnaires within three different samples, the following analysis techniques were used: propensity score matching and linear mixed modeling. Given that within the inpatient setting OQ-45 and BSI data were collected in two different samples, we matched sample 1 and sample 2 using the propensity score technique proposed by Rosenbaum and Rubin [33]. It is primarily used in nonrandomized studies in order to build equivalent samples for causal inference, but it can also be applied to compare different survey samples [34]. In our analysis treatment modalities were defined as the two different questionnaires presented to the patient (BSI versus OQ45 ), and outcome was defined as the scale scores measured at intake and at discharge and then converted either in categories of clinical significance or in $z$ values. As a matching algorithm we applied the nearest neighbor matching with a logistic regression-based propensity score [35]. The following covariates were used in the regression equation: sex, age, marital status, educational level, GAF and CGI, respectively, at intake and at discharge, principal diagnosis, compulsory treatment order, duration of treatment, and type of discharge. Matches were formed on a 1:1 ratio within a caliper size of 0.1 standard deviations of the propensity score. The adequacy of the matching procedure was checked using graphical visualizations of the propensity score distribution; the quality of the balance in the matched groups was examined through the standardized difference of means of each covariate [36, 37]. 


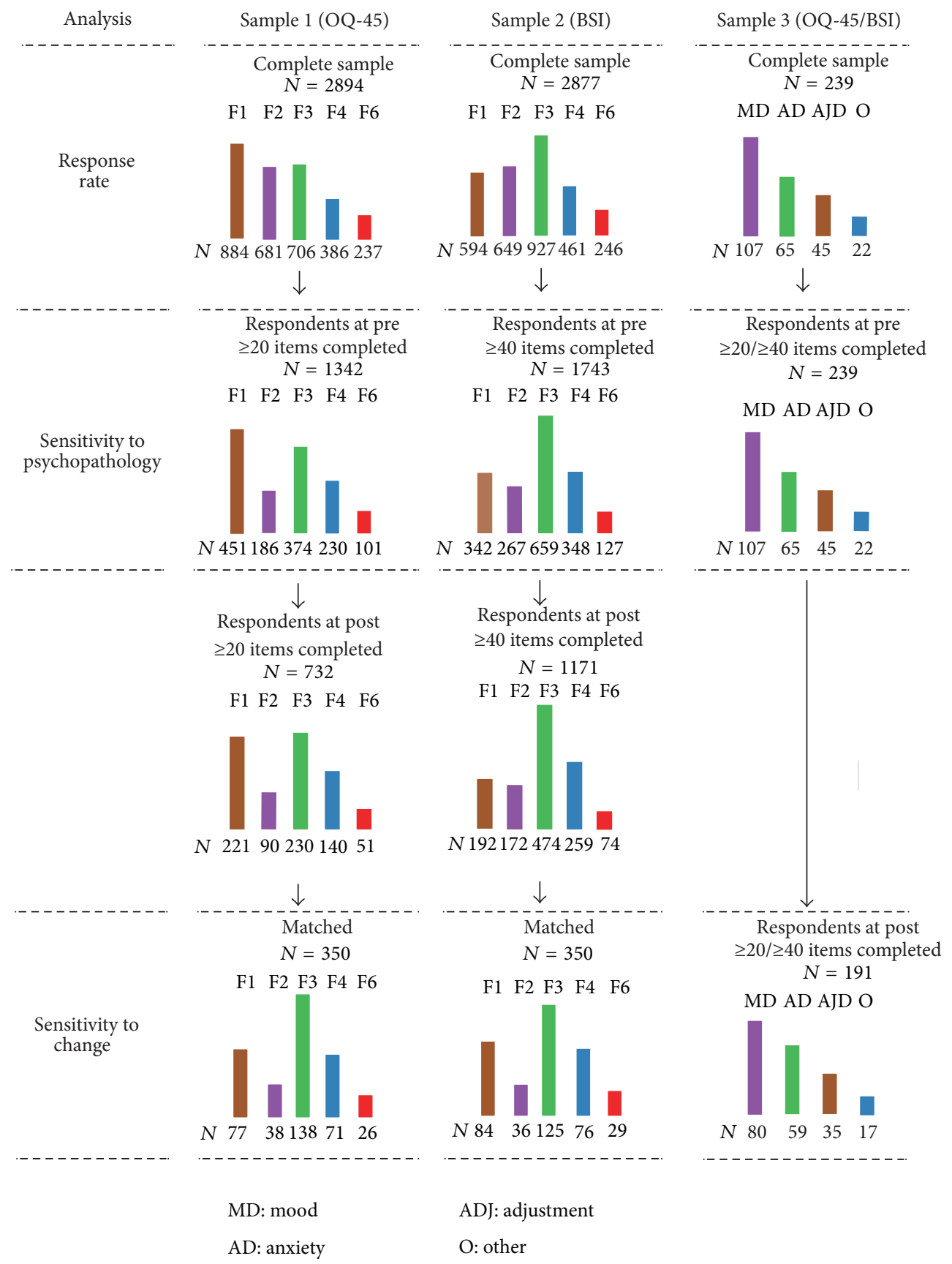

Figure 1: Number of cases included in the different analyses.

Besides the comparison of the proportions of cases in the principal categories of clinical significance classified by the two instruments, we also analyzed the pre-post differences using the linear mixed model; for this, the OQ-45 and BSI scores were $z$-transformed.

\section{Results}

3.1. Response Rate. The completeness of the returned questionnaires varied between the two measures. The highest number of unanswered items was found for the OQ-45 questionnaires returned by the inpatients. Questions on intimate relationships but also work-related questions were the most affected by nonresponse in sample 1. Item number 7 ("I feel unhappy in my marriage/significant relationship") was left out in $24 \%$ of the cases. A work-related item such as number 12 ("I find my work/school satisfying") had $8 \%$ missing values. Overall, the impact of incomplete responses in sample 1 was high: of the 1342 questionnaires evaluable at intake only 588 (43.8\%) had been completely filled out.

Nonresponse on the BSI questionnaires returned by inpatients was not related to the content but instead to the 
TABLE 2: Descriptive statistics and reliability (Chronbach's alpha) of the global scales.

\begin{tabular}{lcccc}
\hline \multicolumn{3}{c}{ OQ Total Score } & & GSI \\
& Inpatients $\left(n_{1}=1342\right)$ & Outpatients $\left(n_{3}=239\right)$ & Inpatients $\left(n_{2}=1743\right)$ & Outpatients $\left(n_{3}=239\right)$ \\
\hline M (SD) & $78.4(28.9)$ & $72.0(19.4)$ & $1.46(0.79)$ & 0.26 \\
Min & 0 & 33 & $0.53)$ & 0.34 \\
Percentiles & & & & \\
5 & 31 & 40 & 0.41 & 0.45 \\
10 & 38 & 47 & 0.85 & 0.66 \\
25 & 58 & 60 & 1.40 & 0.97 \\
50 & 79 & 71 & 2.02 & 1.39 \\
75 & 99 & 85 & 2.56 & 1.76 \\
90 & 117 & 95 & 2.85 & 2.02 \\
95 & 126 & 104 & 3.79 & 2.78 \\
Max & 159 & 143 & 0.97 & 0.95 \\
$\alpha^{*}$ & 0.95 & 0.91 & & \\
\hline
\end{tabular}

Note: ${ }^{*}$ Calculations of $\alpha$ are based on complete case analysis. OQ- 45 from sample 1: $n_{1}=588$. OQ-45 from sample 3: $n_{3}=198$. BSI from sample 2: $n_{2}=1282$. BSI from sample 3: $n_{3}=237$.

TABLE 3: Statistics of the OQ-45 and the BSI/SCL-90R based on samples from Germany.

\begin{tabular}{lccccc}
\hline & \multirow{2}{*}{$\begin{array}{c}\text { Functional population } \\
\text { M (SD) }\end{array}$} & \multicolumn{2}{c}{ Dysfunctional population } & Cutpatients \\
& & M (SD) & Inpatients & M (SD) & Critical difference \\
\hline OQ Total Score & $46.2(18.5)[20]$ & $71.8(21.9)[21]$ & $79.0(27.9)[18]^{*}$ & $58.8[18]$ & $17.8[18]$ \\
GSI & $0.31(0.23)[22]$ & $1.08(0.63)[23]$ & $1.47(0.68)[24]^{\dagger}$ & $0.60[24]^{\dagger}$ & $0.2[25]$ \\
\hline
\end{tabular}

Note: ${ }^{*}$ Pooled M and SD from the intervention and control group at admission. ${ }^{\dagger}$ Based on SCL-90R data.

position of the items. Items 1 to 10 had on average $1.5 \%$ missing values; items above number 40 reached missingness from $4 \%$ to $7.5 \%$. Of the 1743 evaluable BSI forms at intake only $1282(73.6 \%)$ were complete.

In the outpatient sample the incompleteness of the questionnaires was low. For the OQ-45, besides a $7 \%$ of nonresponse on item number 7 , missingness varied from $0 \%$ to $3.5 \%$. On the BSI questionnaires, missingness on the single items was always under $1 \%$.

In the inpatient setting the response rate at both pre- and postmeasurement was about $25 \%$ for the OQ- 45 in sample 1 and $40 \%$ for the BSI in sample 2 . This difference has to be viewed as improvement in data monitoring over the course of the years and cannot be considered to be an indicator for better acceptance of the BSI over the OQ-45.

The response rate was dependent on the severity of psychopathology at admission and of the amount of improvement at discharge in both inpatient samples (Figure 2). Moreover, patients with schizophrenia showed the lowest response rate, with the BSI, $41 \%$ at intake and $45 \%$ at discharge. The highest rate was registered among patients having an F4 diagnosis, with $75 \%$ at intake and $65 \%$ at discharge, respectively.

In the outpatient sample the response rate at the end of the therapy did not correlate significantly with Axis I or Axis II diagnoses.
3.2. Cut-Off and Critical Difference. Table 2 shows the descriptive statistics of the scales based on data collected at the beginning of the treatment in the three samples. Table 3 reports comparative statistics from German sample 3.

The cut-off and the critical difference for the OQ Total Score were calculated using the data of the respondents from sample 1 at intake $\left(N=1342, M_{\text {patient }}=78.4, \mathrm{SD}_{\text {patient }}=28.9\right.$, and Chronbach's $\alpha=0.95)$ and data published by Lambert et al. [20] on a nonclinical sample $\left(N=232, M_{\text {nonpatient }}=\right.$ 46.2, and $\mathrm{SD}_{\text {nonpatient }}=18.5$ ). Applying (1) and (2) led to $C=$ 59 and $D_{95 \%}=18$. Therefore, a patient can be classified as remitted if the following two conditions are fulfilled: (a) his OQ Total Score has decreased by at least 18 points and (b) has reached a value below 59 at the end of the treatment. If only (b) is met, then the patient is classified as improved. Both parameter values are similar to those published in Puschner et al. [18].

The calculation of the parameters for the GSI scale was based on data of the respondents from sample $2(N=1743$, $M_{\text {patient }}=1.46, \mathrm{SD}_{\text {patient }}=0.79$, and Chronbach's $\left.\alpha=0.97\right)$ and the data published in Franke [22] on a nonclinical sample $\left(N=600, M_{\text {nonpatient }}=0.31\right.$, and $\left.\mathrm{SD}_{\text {nonpatient }}=0.23\right)$. The corresponding parameters of clinical significance were $C=$ 0.57 and $D_{95 \%}=0.38$. Schmitz et al. [24] obtained a similar cut-off value based on a sample of participants with severe impairments in which inpatient treatment was indicated. 

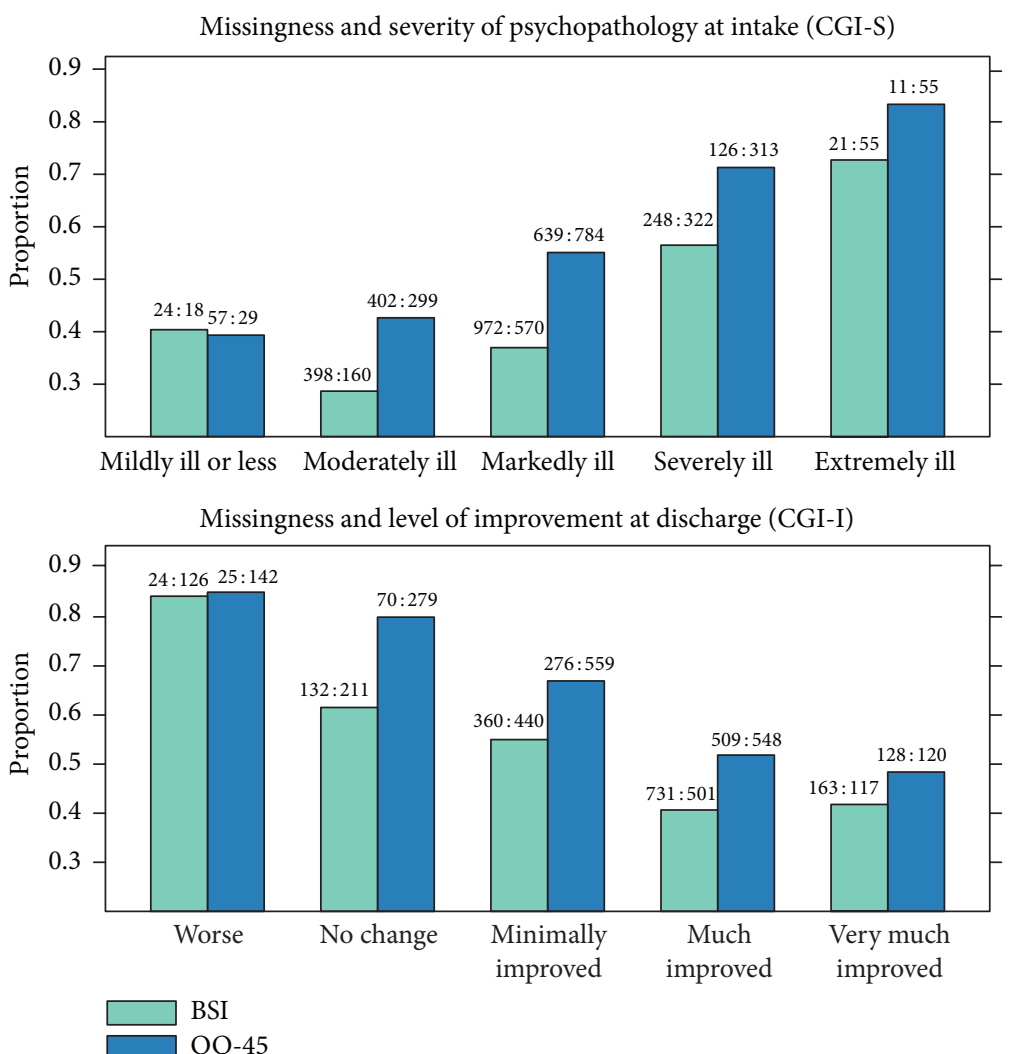

FIGURE 2: Relationship between missingness (proportion of nonresponse) and CGI ratings. The ratio between the number of respondents and the number of nonrespondents is noted on top of the bars (data from samples 1 and 2).

Lutz et al. [25] proposed a critical difference calculated with the standard deviation of the functional sample, which is consequently narrower than our value based on a commonly larger standard deviation from a clinical sample.

3.3. Sensitivity to Psychopathology. Figures 3 and 4 report the sensitivity of the global scales OQ Total Score and GSI. Figure 3 shows the profiles of different diagnosis groups on the OQ-45 scales. More than $80 \%$ of the inpatients with diagnoses F3, F4, and F6 had an OQ Total Score of at least 59 points and were therefore classified as clinical cases (caseness). Fewer than $70 \%$ of inpatients with a diagnosis F1 or F2 scored above the clinical cut-off. Considering the most important clinical disorders in an outpatient setting, that is, affective and anxiety disorders, fewer than $70 \%$ of the patients with an anxiety disorder but without a comorbid personality disorder (PD) were correctly classified as clinical cases by OQ Total Score. In contrast, sensitivity of more than $80 \%$ was attained among patients with an affective disorder, independently of additional comorbidity on the Axis II. Since the SD scale contributes the most to the Total Score scale, a high correlation of $r=0.96$ between the two scales was present. The other two scales, that is, IR and SR, which have a minor importance in forming the Total Score, also did not vary substantially across the different type of disorders.

In contrast to the OQ-45, the BSI produced profiles with more distinctive differences between the diagnosis groups
(Figure 4). Except for the patients with schizophrenia (F2), the Depression scale was the scale with the highest score for the different diagnosis groups. The largest mean on this scale was exhibited by patients with a personality disorder (F6) and not, as theoretically expected, by patients with an affective disorder (F3). Patients with schizophrenia attained the highest score on the Paranoid Ideation scale, which would be in accordance with the diagnostic criteria for this psychiatric group. However, their mean score was outperformed by that of patients with an F6 diagnosis. Overall, the highest Symptom Distress as measured by the BSI was observed on average among patients with a personality disorder in both an inpatient and an outpatient setting.

In the samples of inpatients and outpatients, the BSI with its GSI scale exhibited a higher sensitivity to psychopathology than the OQ-45 with its Total Score scale. However, the same diagnosis groups with a low average OQ Total Score, that is, F2 and F1 among the inpatients and anxiety disorders without PD among the outpatients, achieved a GSI score that was on average also lower than that of other diagnosis groups. About 25\% of the respondents with an F2 diagnosis were not correctly classified as clinical cases. The caseness determined by the GSI scale was not associated with the CGI or GAF score (logistic regression: $\chi^{2}(2)=0.56, p=0.76$ ). Therefore, the substantial misclassification of patients with schizophrenia cannot be considered to be a consequence of low severity of 

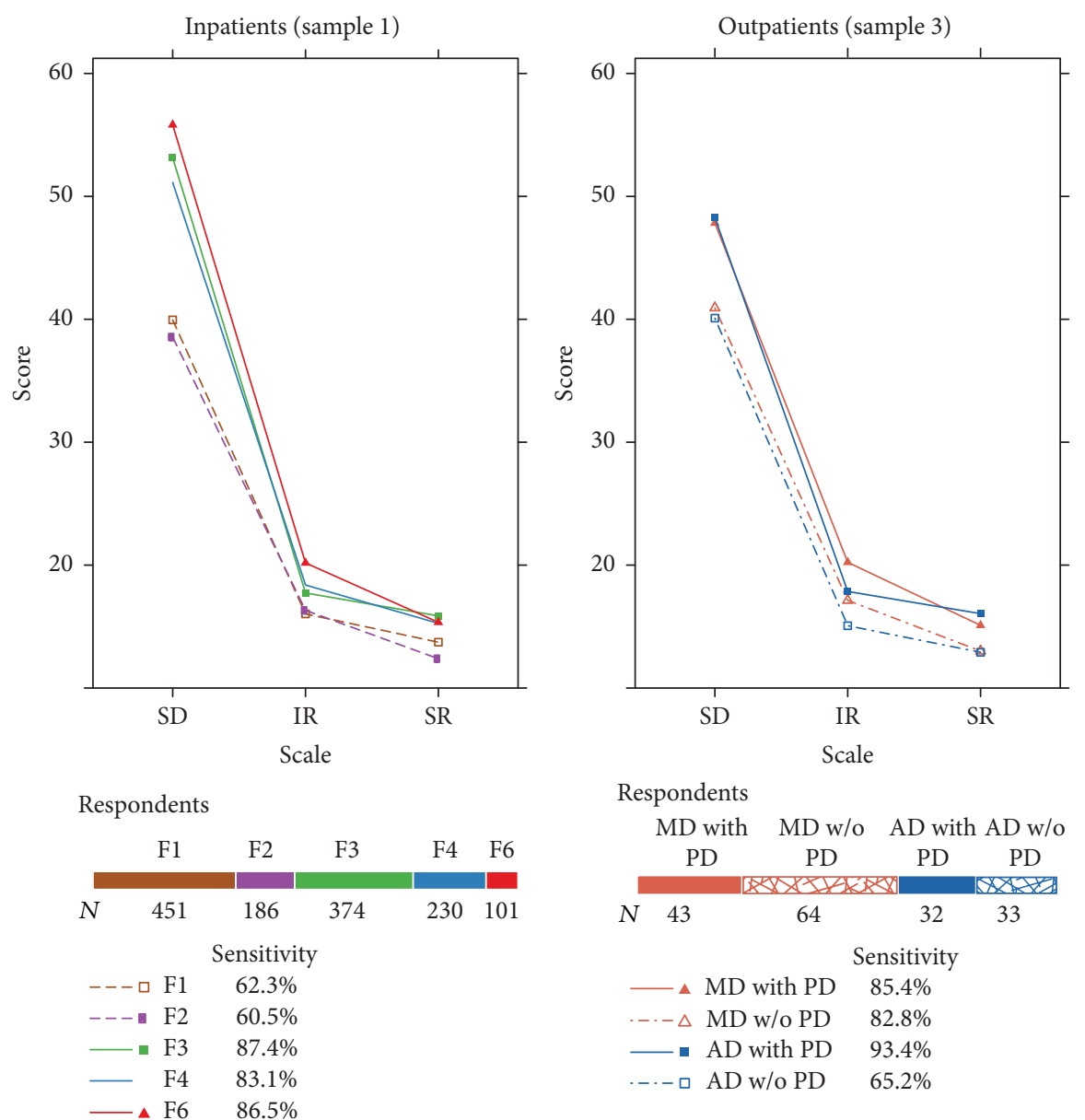

FIGURE 3: OQ-45 profiles of the respondents at intake. For sample 3 only data of patients with a principal diagnosis of mood (MD) or anxiety disorder $(\mathrm{AD})$ grouped according to the presence or absence of a personality disorder (PD) are represented. Percentages represent the sensitivity to psychopathology according to the OQ Total Score.

mental illness or high psychosocial functioning of the sample analyzed.

For both instruments one would actually expect to find higher sensitivity to psychopathology with inpatients than with outpatients, but the percentages in Figures 3 and 4 do not support this expectation. The statistics in Table 2 show that respondents with 0 points on one of the questionnaires were found among the inpatients but not among the outpatients and that the 5th and the 10th percentiles in the inpatient samples were lower than the corresponding values from the outpatients. The lowest 5th percentiles of the global questionnaire scores were found for the GSI among the inpatients with substance abuse (0.15) and inpatients with schizophrenic disorders (0.11).

3.4. Sensitivity to Change. For the inpatient setting, we based our analysis of sensitivity to change on matched samples. The histograms in Figure 5 show that samples 1 and 2 were already quite similar before the matching, since they exhibited an extensive overlap of their propensity score distributions but with some density differences, however. From the smaller sample, that is, sample 1, a total of 419 patients with complete scales and covariates values were available for the matching. Of these, 350 cases could successfully be matched on a $1: 1$ ratio with cases from the larger sample 2 (the absolute standardized difference of means was smaller than 0.1 for each covariate).

Figure 6 shows the results of the clinical significance analysis. In the outpatient sample, the proportions of improved and remitted cases classified by the two measures were quite similar. In the inpatient samples, the GSI scale identified 54\% improved cases compared to the $41.4 \%$ identified by the OQ Total Score. Although this difference is significant $\left[\chi^{2}(7)=\right.$ 58.5, $p<0.001]$, it does not necessarily attest a superior sensitivity to change to the BSI. This is because the GSI scale produces higher prescores than the OQ Total Score, and, therefore, patients have a higher probability of lowering their score on the GSI than on the OQ total scale during their treatment.

To balance out this difference, the scores of the two questionnaires were $z$-transformed and analyzed with a linear mixed model. Figure 7 shows the estimated fixed 

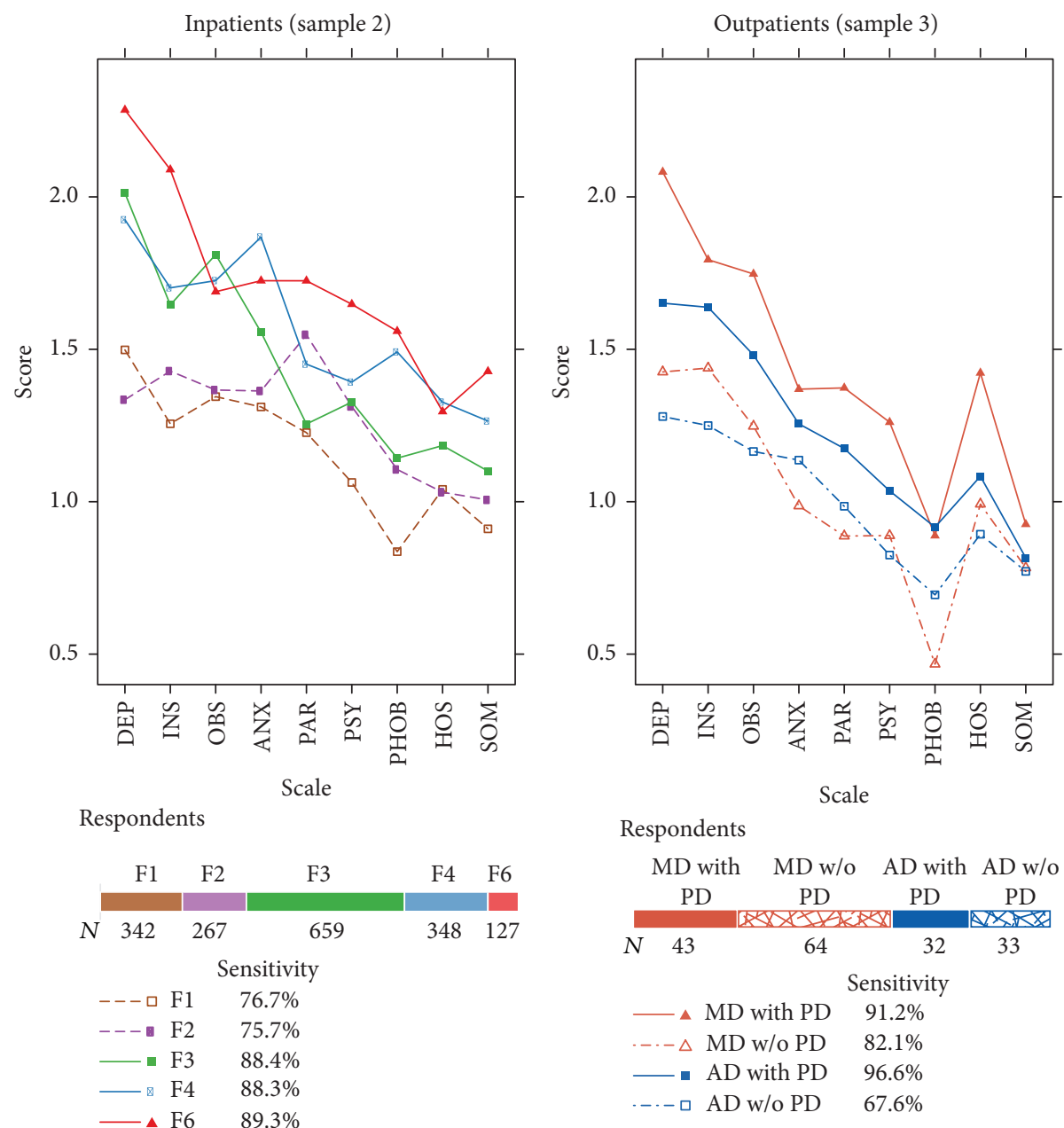

FIGURE 4: BSI profiles of the respondents at intake. For sample 3 only data of patients with a principal diagnosis of mood (MD) or anxiety disorder $(\mathrm{AD})$ grouped according to the presence or absence of a personality disorder (PD) are represented. Percentages represent the sensitivity to psychopathology according to the GSI scale.

effects; they indicate that the two questionnaires recorded the same amount of change between the pre- and the postmeasurement.

\section{Discussion}

This study examined the applicability of the OQ-45 and the BSI for assessing the outcome quality of inpatient and outpatient treatments. Both of these self-report measures can be easily administered by a wide range of service professionals and take about 10 minutes to be filled out. Normative data and analysis results concerning their psychometric properties are available [38].

However, our analyses pointed out the following critical aspects of the performance of the two questionnaires, which have often been neglected in the literature: (1) the number of missing values that emerges in the data collection, (2) the diagnostic value of the scale profiles, and (3) the robustness of the clinical significance algorithm.

(1) Missing Data. Since Rubin's [39] seminal paper on inference with missing data, there has been a growing awareness of this problem in the scientific community. In evaluation studies, the probability of nonresponse is often correlated with the attained outcome. Our results clearly demonstrate this relationship. Respondents and nonrespondents are different in two clinically crucial aspects: nonrespondents have higher severity of mental illness and show less improvement after the treatment than respondents. Missingness is therefore a source of bias when assessing the effectiveness of a treatment, and nowadays guidelines concerning the statistical analysis of incomplete data are available [40,41]. Different authors have pointed out that missingness as low as $10 \%$, if not treated adequately in the statistical analysis, can lead to bias $[42,43]$. In a previous analysis we were able to successfully apply multiple imputation to the outcome data of sample 3 , because, first, the missing rate in the sample was relatively low (20\%) and, second, the progress of the patients was additionally monitored through repeated measurements during the treatment, which generally improve the predictive power of the imputation model [27]. In contrast, with samples 1 and 2 the application of multiple imputation proved to be ineffective. On the one hand, nonresponse in these samples exceeded 50\%, and according to Rubin [44] multiple 
Raw sample 1

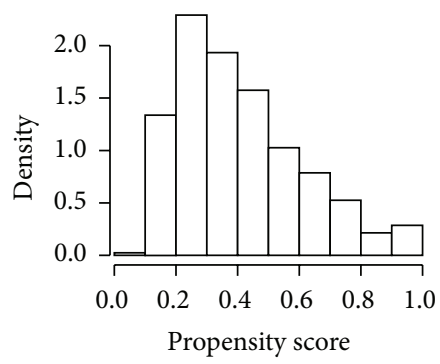

Raw sample 2

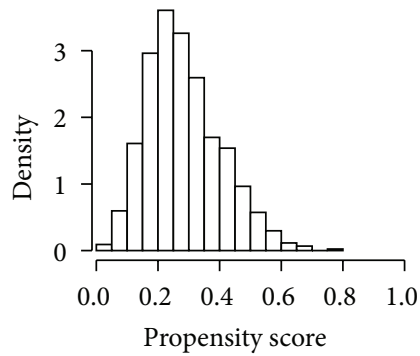

Matched sample 1

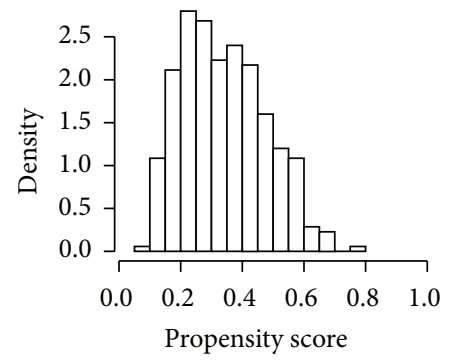

Matched sample 2

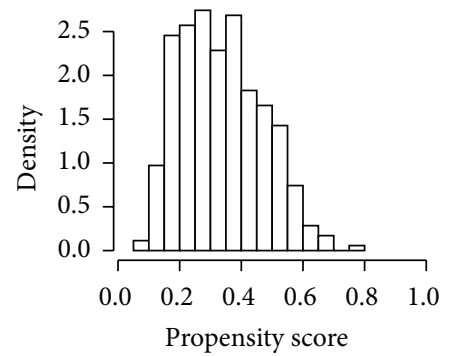

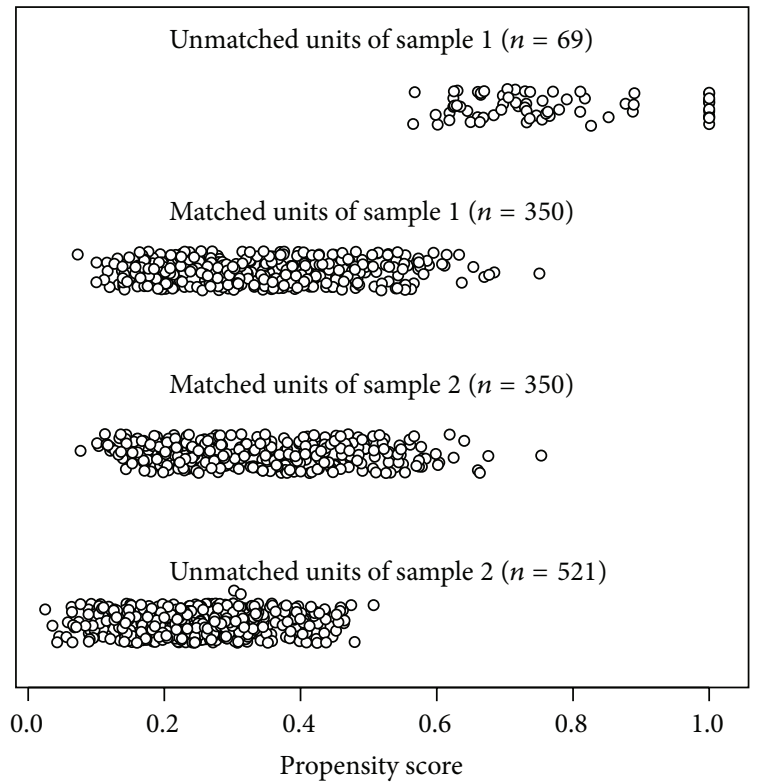

FIGURE 5: Distributions of the propensity scores of the inpatient respondents with complete covariates values. Histograms on the left show the distributions before (raw) and after (matched) the matching. The plot on the right shows the differences between matched and unmatched cases.

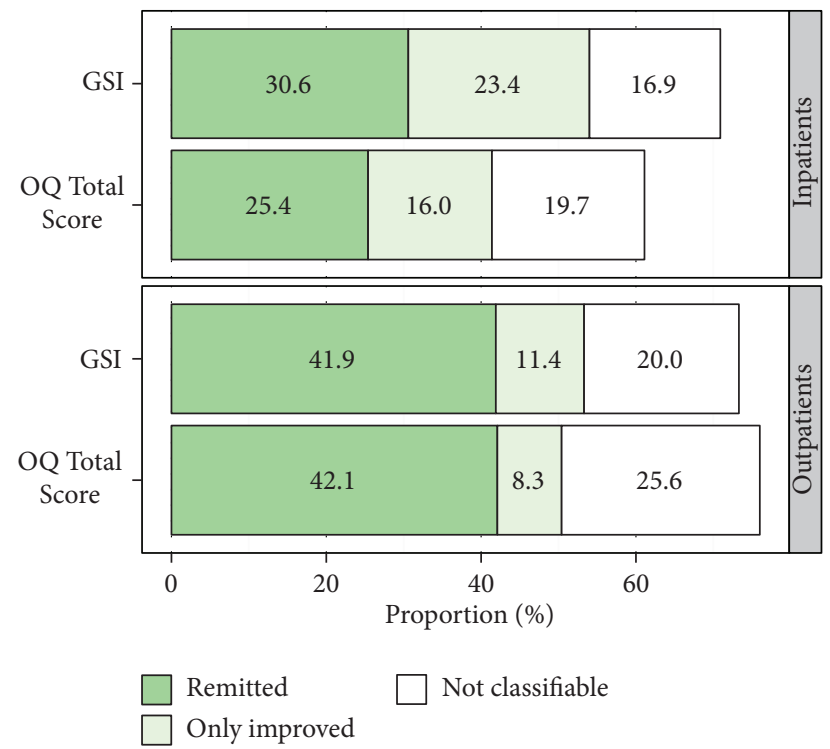

FIGURE 6: Results of the analysis of the clinical significance. Analysis of inpatient data are based on matched samples: $n_{1}=n_{2}=350$. Outpatient sample: $n_{3}=191$. The category "not classifiable" refers to cases misclassified as nonclinical by the self-report measure.

imputation was conceived to deal with a typical fraction of missing information of $30 \%$ or less. On the other hand, the drastic reduction of the data collection to only pre-post measurements, in order to minimize administrative expense, makes it hard to obtain robust estimations of the missing data through imputation models. All in all, the high proportion of missing data discourages the use of self-report measures

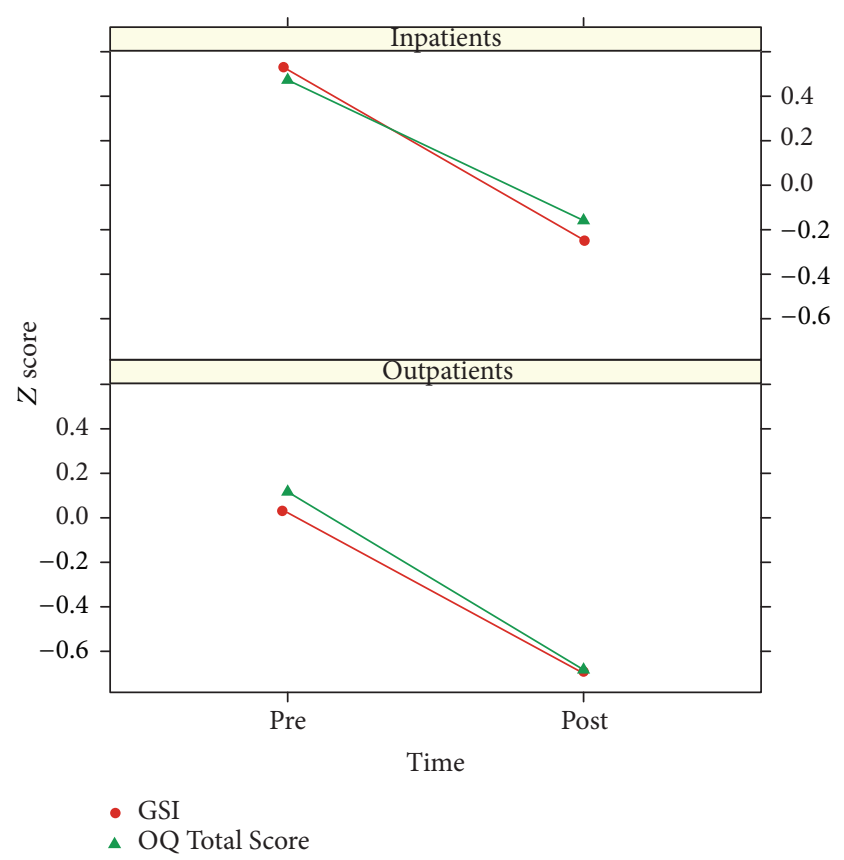

FIgURE 7: Average pre-post changes in $z$ scores. Analysis of inpatient data are based on matched samples: $n_{1}=n_{2}=350$. Outpatient sample: $n_{3}=191$.

with the patients with severe impairments usually found in a psychiatric hospital setting in favor of clinician-administered measures.

(2) Diagnostic Value of the Profiles. Questionnaires like OQ45 and BSI have a relatively large number of items, so that 
different reliable Likert scales may be formed that allow the creation of a person's profile. Can these profiles be used, for instance, to facilitate the formulation of a psychiatric diagnosis? Our results do not support the use of these questionnaires as screening instruments to facilitate the assessment of ICD-10 diagnoses. In the construction of the OQ-45 this was never an intended purpose [45], but the nine primary symptom dimensions of the BSI suggest a possible application for screening purposes. In our sample, inpatients with personality disorders attained on average higher scores than inpatients in other diagnosis groups on six of the nine BSI scales. These patients had a higher mean score on the Depression scale than patients with an affective disorder and a higher mean score on the Paranoid Ideation scale than patients with schizophrenia. Poor diagnostic efficacy of the nine scales in assessing DSM-IV symptom disorders was also reported by Pedereen and Karterud [46]. Two different approaches have been suggested for dealing with the low discriminant validity of the BSI scales. The first is to consider the questionnaire through its GSI score as more appropriate for measuring the overall degree of psychological distress instead of the precise nature of the psychopathology [38]. From this optic, the Outcome Questionnaire with 45 items in its full version or 30 items in its short version would seem to be a more time-effective choice than BSI when measuring general level of psychological distress in a less time-consuming way. The second approach consists in improving the factorial structure of the questionnaire. To this purpose, different authors have used the bifactor structural model in recent years. This model is used to build a general distress factor and more specific components of psychopathology. Thomas [47] demonstrated that a bifactor model of the BSI items can achieve higher accuracy than an oblique simple structure in diagnosing some disorders, such as depression or generalized anxiety disorder. Brodbeck et al. [48] also proposed a bifactor solution that correlates with DSM-IV diagnoses, especially depressive disorders, anxiety disorders, and personality disorders. One of their results in line with ours is that patients with personality disorders are characterized by a high level of general distress. Overall, it seems that improving the factor structure of the BSI can lead to improved sensitivity in identifying depressive or anxiety disorders, but we doubt that it can do the same with patients with substance abuse or acute psychotic disorders. A nonnegligible part of these patients tends to score low, and their profiles resemble those of healthy persons or remitted patients. These results support the hypothesis that patients with these disorders are inclined to underestimate their own emotional and behavioral difficulties. As pointed out by Burlingame et al. [8] the disadvantages of using self-report measures with inpatients with severe and persistent mental illness "include an insufficient clinical picture as a result of the dependence on patients' ability to accurately describe their condition, which at times is doubtful because of denial, minimization of symptoms, or responder bias" (p. 448).

(3) Robustness in the Outcome Evaluation. Clinical significance, as originally proposed by Jacobson and Truax [19], is considered, beside pre-post effect sizes, to be a gold standard as a performance indicator in routine outcome monitoring [49]. This approach encompasses two steps: first, identifying the subsample of patients that reached a reliable change and then determining which among them moved outside the range of the dysfunctional population. This method presupposes a valid and consistent distinction between functionality and dysfunctionality already at the onset of the treatment. Therefore, the percentage of cases that can be adequately categorized depends on the sensitivity of the instrument. For self-report questionnaires measuring important mental health problems, the expected sensitivity is of at least $80 \%$ $[50,51]$. Our results reveal a heterogeneous picture in this regard. For inpatients with an F3, F4, or F6 diagnosis the sensitivity to psychopathology of both instruments exceeds $80 \%$. However, the questionnaires exhibited low values with inpatients with a principal diagnosis of substance abuse or schizophrenia. Especially within the latter group, which represents an important diagnosis group in an inpatient setting, about $25 \%$ of the respondents were misclassified as nonclinical subjects according to the GSI score. The patients with schizophrenia analyzed in this study had a mean GAF score of 32 points at admission and were hospitalized on average 36 days in a primary care hospital. Thus, the substantial misclassification cannot be explained by a lack of mental illness but instead by limitations, either of the measures used in assessing psychopathology or of the methodology used in assessing caseness. Analyses based on outpatients have reported sensitivity of above $90 \%$ for the OQ total scale [52] as well as the GSI scale [46]. Sensitivity values of at least $80 \%$ based on inpatient data were reported by Moessner et al. [53] for both global scales using data from inpatients with an F3, F4, F5, or F6 diagnosis and by Timman et al. [54] for the OQ Total Score using an inpatient sample with personality disorders. Nevertheless, our analyses do not support a generalization of this high accuracy in detecting caseness among inpatients with an F1 or F2 diagnosis. A lack of research on the generalizability of the OQ-45 was confirmed by Lambert and Hawkins [55], who admitted that much of the research "demonstrating the use of the OQ-45 has been conducted with young, educated patients" (p. 496).

Concerning the limitations of our methodology in assessing clinical significance, we see the following possibilities for improvement: (1) construct psychological functioning scales using a bifactor model. For both questionnaires, there are various published results demonstrating that this kind of factor analysis is able to improve the fit of the scale structure to the data $[47,48,56] ;$ (2) determine the cut-off scores with the receiver operating characteristic (ROC) curve. This method can provide more accurate cut-off scores than the weighted midpoints calculated by the Jacobson and Truax method, especially when data are not normally distributed [57]. The procedure requires raw data from both a healthy and a patient sample; however, (3) avoid the necessity of cutoff scores between functionality and dysfunctionality by using the percentage of improvement approach. A different outcome evaluation that could be applied as a complement to the Jacobson and Truax method consists in analysis of the relative change from the baseline severity [58]. A reduction of at least $50 \%$ of the initial symptom level can be considered as 
response to treatment, whereas a reduction of at least $75 \%$ is necessary to rate the outcome as remission [59].

Concerning the comparison of the two outcome instruments, the overall better accuracy of BSI in detecting clinical cases that emerged in our analyses means that patients' prescores are on average lower with the OQ-45 than with the BSI questionnaire. Patients with low scores already at the beginning obviously have on average a minor probability of further lowering their scores during the treatment, independently of the quality of the treatment. Consequently, this disparity between the two measures leads to a higher sensitivity to change for the BSI.

\section{Conclusion}

On the whole, the comparison of the two questionnaires, BSI and OQ-45, as instruments to be used for routine outcome assessment leads to the following statements:

(i) In an inpatient setting both questionnaires have basically the same sensitivity to change. However, the OQ45 has a lower sensitivity to psychopathology than the BSI, a characteristic that also has an impact on sensitivity to change. Another drawback affecting the OQ45 is that inpatients tend to leave out the questions on intimate relationships or work. Unfortunately, in the inpatient setting, the nonresponse rate with both self-report measures is higher than $30 \%$, leading to potentially nonrepresentative results.

(ii) In an outpatient setting, the superiority of the BSI in both types of sensitivity is minimal. Therefore, OQ45 can be considered as an equivalent alternative to the BSI in routine outcome monitoring, with the advantage that it is less time-consuming.

(iii) Due to the limited sensitivity to psychopathology in both treatment settings, it is not advisable to base clinical assessment on data collected only with these selfreport questionnaires; they should be complemented with clinician-completed ratings.

\section{Competing Interests}

The authors declare that they have no competing interests.

\section{References}

[1] APA Task Force on Quality Indicators, Quality Indicators. Defining and Measuring Quality in Psychiatric Care for Adults and Children. Report of the APA Task Force on Quality Indicators and Report of the APA Task Force on Quality Indicators for Children, American Psychiatric Publishing, Washington, DC, USA, 2002.

[2] B. M. Ogles, M. J. Lambert, and S. A. Fields, Essentials of Outcome Assessment, John Wiley \& Sons, New York, NY, USA, 2002.

[3] A. J. Rush, M. B. First, and D. Blacker, Handbook of Psychiatric Measures, American Psychiatric Publishing, Washington, DC, USA, 2008.
[4] T. Wobrock, S. Weinmann, P. Falkai, and W. Gaebel, "Quality assurance in psychiatry: quality indicators and guideline implementation," European Archives of Psychiatry and Clinical Neuroscience, vol. 259, no. 2, pp. S219-S226, 2009.

[5] L. R. Derogatis and N. Melisaratos, "The brief symptom inventory: an introductory report," Psychological Medicine, vol. 13, no. 3, pp. 595-605, 1983.

[6] L. R. Derogatis, SCL-90-R Revised Manual, Johns Hopkins School of Medicine, Baltimore, Md, USA, 1983.

[7] T. L. Kramer and G. R. Smith, "Behavioral health outcomes," in Textbook of Administrative Psychiatry: New Concepts for a Changing Behavioral Health System, J. A. Talbott and R. E. Hales, Eds., pp. 135-144, American Psychiatric Publishing, 2001.

[8] G. M. Burlingame, T. W. Dunn, S. Chen et al., "Special section on the GAF: selection of outcome assessment instruments for inpatients with severe and persistent mental illness," Psychiatric Services, vol. 56, no. 4, pp. 444-451, 2005.

[9] H. L. Piersma, W. M. Reaume, and J. L. Boes, "The Brief Symptom Inventory (BSI) as an outcome measure for adult psychiatric inpatients," Journal of Clinical Psychology, vol. 50, no. 4, pp. 555-563, 1994.

[10] M. Hoe and J. Brekke, "Testing the cross-ethnic construct validity of the brief symptom inventory," Research on Social Work Practice, vol. 19, no. 1, pp. 93-103, 2009.

[11] W. D. Wood, "An attempt to validate the psychoticism scale of the brief symptom inventory," British Journal of Medical Psychology, vol. 55, no. 4, pp. 367-373, 1982.

[12] M. E. Johnson, C. L. Chipp, C. Brems, and D. B. Neal, "Receiver operating characteristics for the brief symptom inventory depression, paranoid ideation, and psychoticism scales in a large sample of clinical inpatients," Psychological Reports, vol. 102, no. 3, pp. 695-705, 2008.

[13] N. J. Preston and T. J. Harrison, "The Brief Symptom Inventory and the Positive and Negative Syndrome Scale: discriminate validity between a self-reported and observational measure of psychopathology," Comprehensive Psychiatry, vol. 44, no. 3, pp. 220-226, 2003.

[14] M. J. Lambert, J. J. Morton, D. Hatfield et al., Administration and Scoring Manual for the OQ-45, American Professional Credentialing Services, Nashville, Tenn, USA, 2004.

[15] M. E. Maruish, Essentials of Treatment Planning, John Wiley \& Sons, New York, NY, USA, 2002.

[16] L. Bufka and N. Camp, "Brief measures for screening and measuring mental health outcomes," in Handbook of Assessment and Treatment Planning for Psychological Disorders, M. M. Antony and D. H. Barlow, Eds., vol. 2, pp. 62-94, Guilford Press, New York, NY, USA, 2010.

[17] L. A. Doerfler, M. E. Addis, and P. W. Moran, "Evaluating mental health outcomes in an inpatient setting: convergent and divergent validity of the OQ-45 and Basis-32," The Journal of Behavioral Health Services and Research, vol. 29, no. 4, pp. 394403, 2002.

[18] B. Puschner, D. Schöfer, C. Knaup, and T. Becker, "Outcome management in in-patient psychiatric care," Acta Psychiatrica Scandinavica, vol. 120, no. 4, pp. 308-319, 2009.

[19] N. S. Jacobson and P. Truax, "Clinical significance: a statistical approach to defining meaningful change in psychotherapy research," Journal of Consulting and Clinical Psychology, vol. 59, no. 1, pp. 12-19, 1991.

[20] M. J. Lambert, W. Hannöver, K. Nisslmüller, M. Richard, and H. Kordy, "Fragebogen zum Ergebnis von Psychotherapie, 
[Questionnaire on the results of psychotherapy: reliability and validity of the German translation of the Outcome Questionnaire 45.2 (OQ-45.2)]," Zeitschrift für klinische Psychologie und Psychotherapie, vol. 31, no. 1, pp. 40-46, 2002.

[21] B. Puschner, S. Haug, S. Häfner, and H. Kordy, "Impact of treatment setting on the course of improvement. Inpatient versus outpatient psychotherapy," Psychotherapeut, vol. 49, no. 3, pp. 182-192, 2004.

[22] G. H. Franke, "Erste Studien zur Güte des Brief Symptom Inventory (BSI) [First studies about the psychometric quality of the brief symptom inventory (BSI)," Zeitschrift für Medizinische Psychologie, vol. 6, pp. 159-166, 1997.

[23] C. Geisheim, K. Hahlweg, W. Fiegenbaum, M. Frank, B. Schroeder, and I. von Witzleben, "Das Brief Symptom Inventory (BSI) als Instrument zur Qualitätssicherung in der Psychotherapie," Diagnostica, vol. 48, no. 1, pp. 28-36, 2002.

[24] N. Schmitz, N. Hartkamp, and G. H. Franke, "Assessing clinically significant change: application to the SCL-90-R," Psychological Reports, vol. 86, no. 1, pp. 263-274, 2000.

[25] W. Lutz, J. R. Böhnke, K. Köck, and A. Bittermann, “Diagnostik und psychometrische Verlaufsrückmeldungen im Rahmen eines Modellprojektes zur Qualitätssicherung in der ambulanten Psychotherapie," Zeitschrift für Klinische Psychologie und Psychotherapie, vol. 40, no. 4, pp. 283-297, 2011.

[26] V. Tschuschke, A. Crameri, M. Koehler et al., "The role of therapists' treatment adherence, professional experience, therapeutic alliance, and clients' severity of psychological problems: prediction of treatment outcome in eight different psychotherapy approaches. Preliminary results of a naturalistic study," Psychotherapy Research, vol. 25, no. 4, pp. 420-434, 2015.

[27] A. Crameri, A. von Wyl, M. Koemeda, P. Schulthess, and V. Tschuschke, "Sensitivity analysis in multiple imputation in effectiveness studies of psychotherapy," Frontiers in Psychology, vol. 6, no. 1042, pp. 1-11, 2015.

[28] P. Staczan, R. Schmuecker, M. Koehler et al., "Effects of sex and gender in ten types of psychotherapy," Psychotherapy Research, 2015.

[29] H. U. Wittchen, M. Zaudig, and T. Fydrich, Structured Clinical Interview for DSM-IV. Axis I and II, Hogrefe, Göttingen, Germany, 1997.

[30] J. Busner and S. D. Targum, "The clinical global impressions scale: applying a research tool in clinical practice," Psychiatry (Edgmont), vol. 4, no. 7, pp. 28-37, 2007.

[31] J. Endicott, R. L. Spitzer, J. L. Fleiss, and J. Cohen, “The global assessment scale. A procedure for measuring overall severity of psychiatric disturbance," Archives of General Psychiatry, vol. 33, no. 6, pp. 766-771, 1976.

[32] G. H. Franke, Brief Symptom Inventory von Derogatis (BSI), Hogrefe, Göttingen, Germany, 2000.

[33] P. R. Rosenbaum and D. B. Rubin, "The central role of the propensity score in observational studies for causal effects," Biometrika, vol. 70, no. 1, pp. 41-55, 1983.

[34] R. Kenett and S. Salini, Modern Analysis of Customer Surveys: With Applications Using R, John Wiley \& Sons, New York, NY, USA, 2011.

[35] D. Ho, K. Imai, G. King, and E. Stuart, "Matchit: nonparametric preprocessing for parametric casual inference," Journal of Statistical Software, vol. 42, no. 8, pp. 1-28, 2011.

[36] E. A. Stuart, "Matching methods for causal inference: a review and a look forward," Statistical Science, vol. 25, no. 1, pp. 1-21, 2010.
[37] D. B. Rubin, "Using propensity scores to help design observational studies: application to the tobacco litigation," Health Services and Outcomes Research Methodology, vol. 2, no. 3-4, pp. 169-188, 2001.

[38] A. M. Tarescavage and Y. S. Ben-Porath, "Psychotherapeutic outcomes measures: a critical review for practitioners," Journal of Clinical Psychology, vol. 70, no. 9, pp. 808-830, 2014.

[39] D. B. Rubin, "Inference and missing data," Biometrika, vol. 63, no. 3, pp. 581-592, 1976.

[40] C. H. Mallinckrodt, Q. Lin, and M. Molenberghs, "A structured framework for assessing sensitivity to missing data assumptions in longitudinal clinical trials," Pharmaceutical Statistics, vol. 12, no. 1, pp. 1-6, 2013.

[41] National Research Council, The Prevention and Treatment of Missing Data in Clinical Trials, The National Academies Press, Washington, DC, USA, 2010.

[42] V. Kristman, M. Manno, and P. Côté, "Loss to follow-up in cohort studies: how much is too much?" European Journal of Epidemiology, vol. 19, no. 8, pp. 751-760, 2004.

[43] A. M. Wood, I. R. White, and S. G. Thompson, "Are missing outcome data adequately handled? A review of published randomized controlled trials in major medical journals," Clinical Trials, vol. 1, no. 4, pp. 368-376, 2004.

[44] D. B. Rubin, "Discussion on multiple imputation," International Statistical Review, vol. 71, no. 3, pp. 619-625, 2003.

[45] M. J. Lambert, Prevention of Treatment Failure: The Use of Measuring, Monitoring, and Feedback in Clinical Practice, American Psychological Association, Washington, DC, USA, 2010.

[46] G. Pedereen and S. Karterud, "Is SCL-90R helpful for the clinician in assessing DSM-IV symptom disorders?" Acta Psychiatrica Scandinavica, vol. 110, no. 3, pp. 215-224, 2004.

[47] M. L. Thomas, "Rewards of bridging the divide between measurement and clinical theory: demonstration of a bifactor model for the Brief Symptom Inventory," Psychological Assessment, vol. 24, no. 1, pp. 101-113, 2012.

[48] J. Brodbeck, N. Stulz, S. Itten, D. Regli, H. Znoj, and F. Caspar, "The structure of psychopathological symptoms and the associations with DSM-diagnoses in treatment seeking individuals," Comprehensive Psychiatry, vol. 55, no. 3, pp. 714726, 2014.

[49] E. de Beurs, M. Barendregt, A. de Heer et al., "Comparing methods to denote treatment outcome in clinical research and benchmarking mental health care," Clinical Psychology \& Psychotherapy, vol. 23, no. 4, pp. 308-318, 2016.

[50] J. J. M. H. Strik, A. Honig, R. Lousberg, and J. Denollet, "Sensitivity and specificity of observer and self-report questionnaires in major and minor depression following myocardial infarction," Psychosomatics, vol. 42, no. 5, pp. 423-428, 2001.

[51] I. Bjelland, A. A. Dahl, T. T. Haug, and D. Neckelmann, "The validity of the Hospital Anxiety and Depression Scale: an updated literature review," Journal of Psychosomatic Research, vol. 52, no. 2, pp. 69-77, 2002.

[52] I. Amble, T. Gude, S. Stubdal et al., "Psychometric properties of the Outcome Questionnaire-45.2: the Norwegian version in an international context," Psychotherapy Research, vol. 24, no. 4, pp. 504-513, 2014.

[53] M. Moessner, C. Gallas, S. Haug, and H. Kordy, "The clinical psychological diagnostic system (KPD-38): sensitivity to change and validity of a self-report instrument for outcome monitoring and quality assurance," Clinical Psychology and Psychotherapy, vol. 18, no. 4, pp. 331-338, 2011. 
[54] R. Timman, K. de Jong, and N. de Neve-Enthoven, "Cutoff scores and clinical change indices for the dutch outcome questionnaire (OQ-45) in a large sample of normal and several psychotherapeutic populations," Clinical Psychology \& Psychotherapy, 2015.

[55] M. J. Lambert and E. J. Hawkins, "Measuring outcome in professional practice: considerations in selecting and using brief outcome instruments," Professional Psychology: Research and Practice, vol. 35, no. 5, pp. 492-499, 2004.

[56] A. G. Thalmayer, "Alternative models of the Outcome Questionnaire-45," European Journal of Psychological Assessment, vol. 31, no. 2, pp. 120-130, 2015.

[57] M. Gönen, Analyzing Receiver Operating Characteristic Curves with SAS, SAS Institute, Cary, NC, USA, 2007.

[58] W. Hiller, A. C. Schindler, and M. J. Lambert, "Defining response and remission in psychotherapy research: a comparison of the RCI and the method of percent improvement," Psychotherapy Research, vol. 22, no. 1, pp. 1-11, 2012.

[59] M. L. Crismon, M. Trivedi, T. A. Pigott et al., “The Texas medication algorithm project: report of the Texas consensus conference panel on medication treatment of major depressive disorder," The Journal of Clinical Psychiatry, vol. 60, no. 3, pp. 142-156, 1999. 


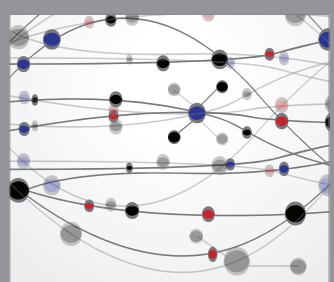

The Scientific World Journal
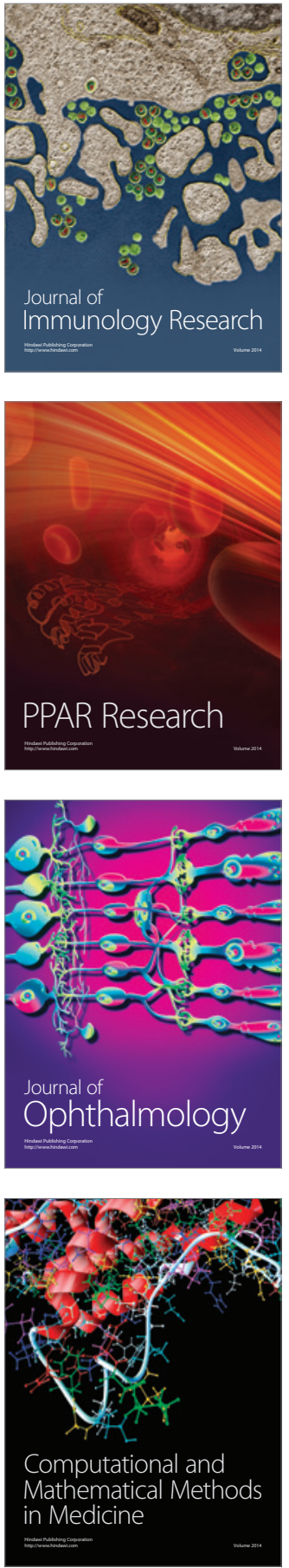

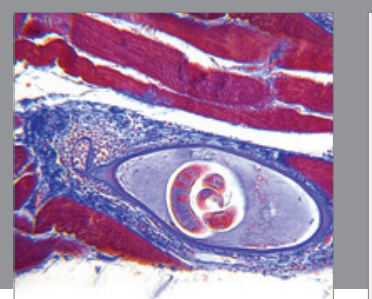

Gastroenterology Research and Practice

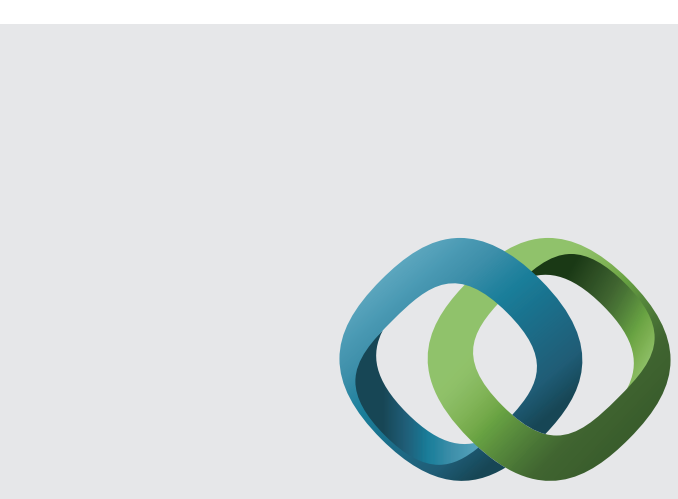

\section{Hindawi}

Submit your manuscripts at

http://www.hindawi.com
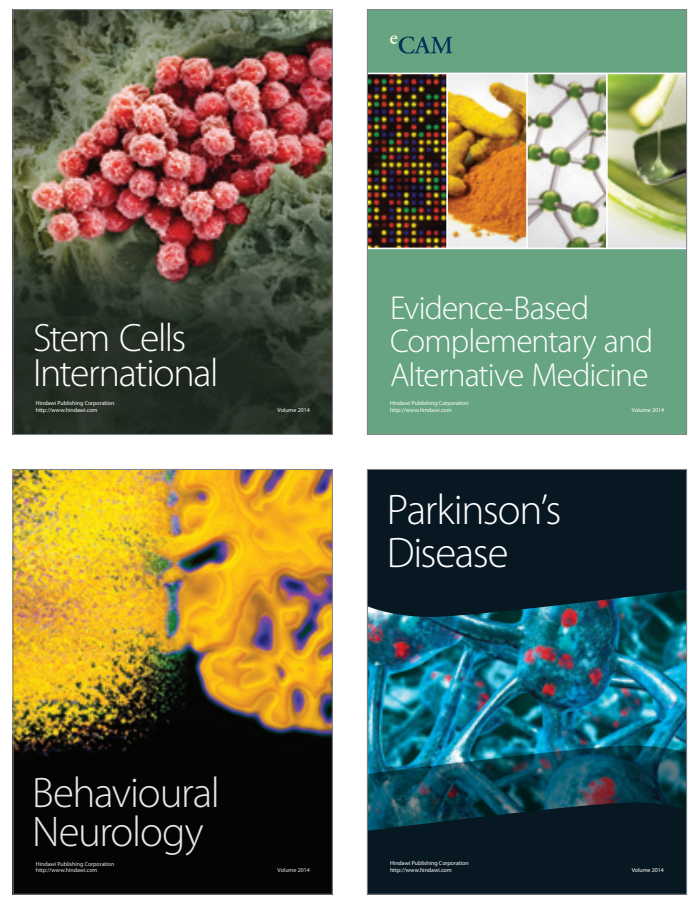
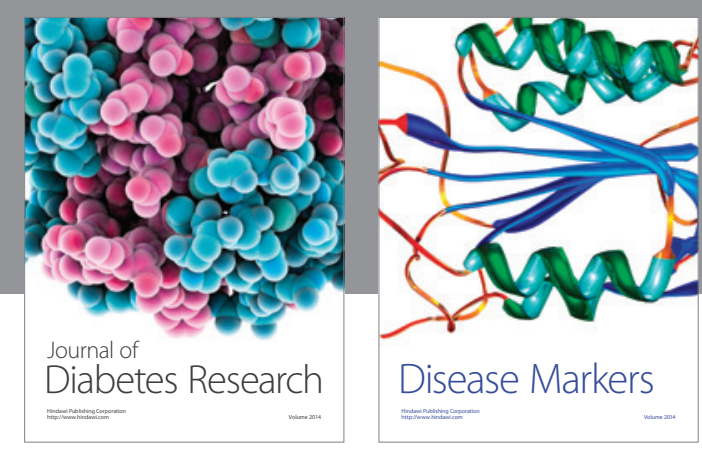

Disease Markers
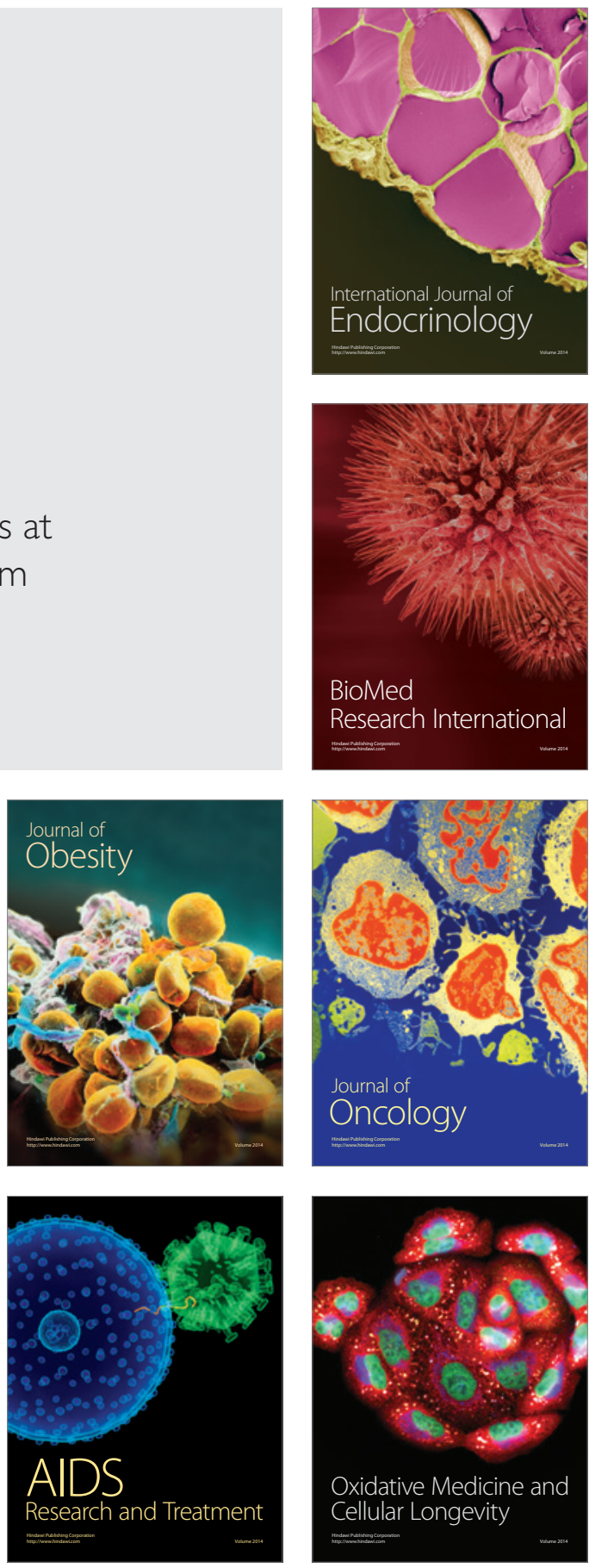